\title{
One-Step, Solvothermal Synthesis of Graphene-CdS and Graphene-ZnS Quantum Dot Nanocomposites and Their Interesting Photovoltaic Properties
}

\author{
Ping Wang ${ }^{1}$, Tengfei Jiang ${ }^{2}$, Chengzhou Zhu ${ }^{1}$, Yueming Zhai ${ }^{1}$, Dejun Wang ${ }^{2}$, and Shaojun Dong ${ }^{1}(\varangle)$ \\ ${ }^{1}$ State Key Laboratory of Electroanalytical Chemistry, Changchun Institute of Applied Chemistry, Chinese Academy of Sciences, \\ Changchun 130022, China \\ ${ }^{2}$ College of Chemistry, Jilin University, Changchun 130012, China
}

Received: 26 August 2010 / Revised: 14 September 2010 / Accepted: 14 September 2010

(C) The Author(s) 2010. This article is published with open access at Springerlink.com

\begin{abstract}
The synthesis of graphene-semiconductor nanocomposites has attracted increasing attention due to their interesting optoelectronic properties. However the synthesis of such nanocomposites, with decorated particles well dispersed on graphene, is still a great challenge. This work reports a facile, one-step, solvothermal method for the synthesis of graphene-CdS and graphene-ZnS quantum dot nanocomposites directly from graphene oxide, with CdS and ZnS very well dispersed on the graphene nanosheets. Photoluminescence measurements showed that the integration of CdS and ZnS with graphene significantly decreases their photoluminescence. Transient photovoltage studies revealed that the graphene-CdS nanocomposite exhibits a very unexpected strong positive photovoltaic response, while separate samples of graphene and CdS quantum dots (QDs) of a similar size do not show any photovoltaic response.
\end{abstract}

\section{KEYWORDS}

Graphene, CdS, ZnS, quantum dots, charge transfer, photovoltaic

\section{Introduction}

Graphene nanosheets (GNs), as a new class of oneatom-thick, two-dimensional carbon material, have been intensively investigated in recent years due to their outstanding electronic, optical and mechanical properties [1,2]. The unique $\mathrm{sp}^{2}$ hybrid carbon nanostructure of GNs opens up new applications in nanoelectronics [3], biosensors [4, 5], supercapacitors [6], and transistors [7]. Owing to their remarkable high electron mobility $\left(15,000 \mathrm{~cm}^{2} / \mathrm{V} \cdot \mathrm{s}\right)$ [8], extremely large surface area $\left(\sim 2600 \mathrm{~m}^{2} / \mathrm{g}\right)$ [9], and low fabrication cost, GNs are considered as an ideal support for developing next-generation photovoltaic devices [10]. $\mathrm{Li}$ et al. reported that on forming layered GNs-CdS quantum dots (QDs), the electrode exhibited a significant improvement in photo-response compared with that of single-walled carbon nanotube-CdS QD nanocomposites [11].

Before the emergence of GNs, some research had already been carried out on the integration of semiconductor QDs (such as CdS and ZnS) and carbon nanotubes, because of the promising electronic and optoelectronic properties of the resulting materials

Address correspondence to dongsj@ciac.jl.cn 
$[12,13]$. However, to date there have been few demonstrations of the synthesis of GNs-semiconductor QD nanocomposites with a high degree of dispersion $[14,15]$. The main hurdle in the synthesis of semiconductor QDs on the surface of GNs is that introducing the metal ions into an aqueous solution of GNs causes their immediate aggregation. Thus, one cannot obtain well dispersed semiconductor QDs on GNs. Herein, we report a facile one-step, solvothermal method for the synthesis of nanocomposites of GNs with metal sulfides such as $\mathrm{CdS}$ and $\mathrm{ZnS}$, with a high degree of dispersion and high coverage, directly from graphene oxide (GO). The photoluminescence (PL) and photovoltaic properties of the as-synthesized GNs-CdS quantum dot nanocomposites were also studied.

The synthesis of GNs-metal sulfide (GNs-MS) nanocomposites is shown in Scheme 1. As illustrated, ethylene glycol (EG), metal acetate salts $M(\mathrm{Ac})_{2}(M=$ $\mathrm{Cd}, \mathrm{Zn}$ ), and thiourea (TU) serve as the solvent, and the source of the metal and sulfur, respectively.

Several benefits motivated us to use EG as the solvent: a) the as-prepared GO could be well dispersed in EG by sonication and remained stable for a long time [16], b) the metal ions can be homogeneously dissolved into EG without inducing the aggregation of GO (Fig. S-1, in the Electronic Supplementary Material (ESM)), which is very important to ensure the homogeneous growth of metal sulfide QDs on the surface of GNs, and c) EG has a strong ability to reduce GO into GNs at high temperatures [17]. It is now known that semiconductor nanoparticles interact with GO through its carboxylic acid functional groups [18]. However, the carboxylic acid functional groups are mainly located at the edge of GO [19] and may not be

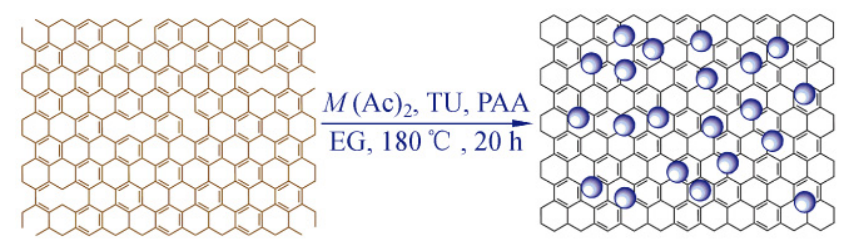

Scheme 1 Schematic illustration of the one-pot synthesis of GNs-MS QD nanocomposites, where $M$ represents $\mathrm{Cd}$ or $\mathrm{Zn}$, Ac is acetate, TU is thiourea, PAA is poly(acrylic acid) and EG is ethylene glycol. The size of the CdS (or ZnS) QDs in the scheme does not correspond to the actual size of the synthesized CdS (or $\mathrm{ZnS}$ ) QDs on GNs sufficient in number to give homogeneous growth of semiconductor nanoparticles on GNs. Thus, we introduced poly(acrylic acid) (PAA), which possesses an abundance of carboxylic acid functional groups, into the reaction system in order to overcome this shortcoming. Control experiments showed that PAA play a key role in the homogenous growth of CdS ( $\mathrm{ZnS})$ QDs on GNs (Fig. S-2, in the ESM).

Low magnification transmission electron microscopy (TEM) images of GNs-CdS and GNs-ZnS QD nanocomposites (Fig. S-3, in the ESM) indicate that after carefully washing the product, hardly any undecorated particles could be observed, and the GNs are well spread out. Further investigations (Figs. 1(a) and 1(c)) show that the CdS and ZnS QDs are well dispersed on the GNs, and no obvious aggregation is observed. It should be noted here that no GN areas not decorated with CdS or ZnS QDs were found, which indicates the high-coverage of the QDs on GNs. Highresolution transmission electron microscopy (HRTEM) images (Figs. 1(b) and 1(d)) show that the sizes of decorated CdS and ZnS QDs are 3-4 and 1-2 nm, respectively. The insets in the HRTEM images indicate that both CdS and ZnS QDs on GNs are well crystallized.

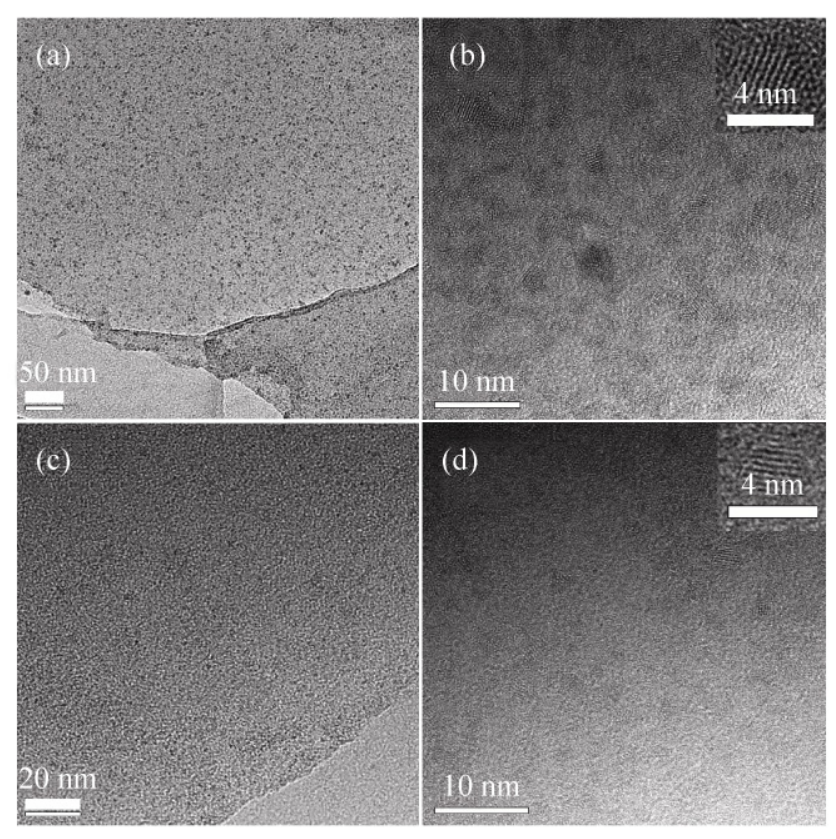

Figure 1 TEM and high-resolution TEM (HRTEM) image of GNs-CdS (a, b) and GNs-ZnS (c, d) QD nanocomposites. The insets in (b) and (d) show HRTEM images of single CdS and $\mathrm{ZnS}$ QDs, respectively 
Energy dispersive X-ray (EDX) analysis (Fig. S-4, in the ESM) further confirms the presence of $\mathrm{Cd}$ (or $\mathrm{Zn}$ ) and $S$ elements in the corresponding GNs-MS nanocomposite. X-ray diffraction (XRD) (Fig. S-5, in the ESM) of GNs-CdS and GNs-ZnS shows that the decorated $\mathrm{CdS}$ and ZnS QDs both have the cubic zinc blende structure (for CdS JCPDS No. 42-1411, for ZnS JCPDS No. 77-2100), and the very broad diffraction peaks are indicative of small particle sizes of $C d S$ and $\mathrm{ZnS}$. The average crystallite sizes of $\mathrm{CdS}$ and $\mathrm{ZnS}$ QDs, calculated from the Scherrer equation are about 4.02 and $2.29 \mathrm{~nm}$, respectively, which are slightly larger than the values given by HRTEM.

X-ray photoelectron spectroscopy (XPS) was used to confirm the formation of graphene from GO during the solvothermal treatment (Fig. 2). Four peaks located at 284.5, 285.6, 286.7, and $288.4 \mathrm{eV}$ observed from the $\mathrm{C} 1 \mathrm{~s}$ deconvolution spectrum of $\mathrm{GO}$ correspond to the $\mathrm{C}-\mathrm{C}, \mathrm{C}-\mathrm{OH}, \mathrm{C}-\mathrm{O}$, and $\mathrm{O}-\mathrm{C}=\mathrm{O}\left(-\mathrm{COO}^{-}\right)$groups, respectively. Compared with $\mathrm{GO}$, the intensity of the $\mathrm{C}-\mathrm{O}$ peak in GNs, GNs-CdS and GNs-ZnS, is dramatically decreased, while the peak intensity due to $\mathrm{C}-\mathrm{OH}$ and $\mathrm{O}-\mathrm{C}=\mathrm{O}$ is increased. The amount of $\mathrm{CdS}$ $(\mathrm{ZnS}) \mathrm{QDs}$ in the GNs-CdS (ZnS) is $33.8 \%(12.7 \%)$, calculated on the basis of the XPS results (Fig. S-6, in the ESM). The results indicate that a) most of the oxygen-containing functional groups were removed after reduction by EG, b) PAA, the surfactant used, was bonded onto the GNs, GNs-CdS, and GNs-ZnS; this was confirmed by Fourier transform infrared (FTIR) spectroscopy (Fig. S-7, in the ESM). Comparison of the FTIR spectra of different samples shows that the band at $1055 \mathrm{~cm}^{-1}$ (C-O stretching vibration) of GO is decreased for GNs, GNs-CdS, and GNs-ZnS, which indicates significant chemical reduction of this group [15]. In addition, the characteristic absorption peak at $\sim 1724 \mathrm{~cm}^{-1}$ ( $\mathrm{C}=\mathrm{O}$ stretching vibration), and the vibration and deformation peaks of $\mathrm{O}-\mathrm{H}$ groups at $\sim 3378 \mathrm{~cm}^{-1}$ and $\sim 1410 \mathrm{~cm}^{-1}$ in the spectra of GNs, GNs-CdS, and GNs-ZnS demonstrate the presence of the bonded PAA. The reduction of GO during the reaction is also confirmed by the blue shift of the G-band (Fig. S-8, in the ESM) in the Raman spectra [15].

Due to the promising charge transport properties
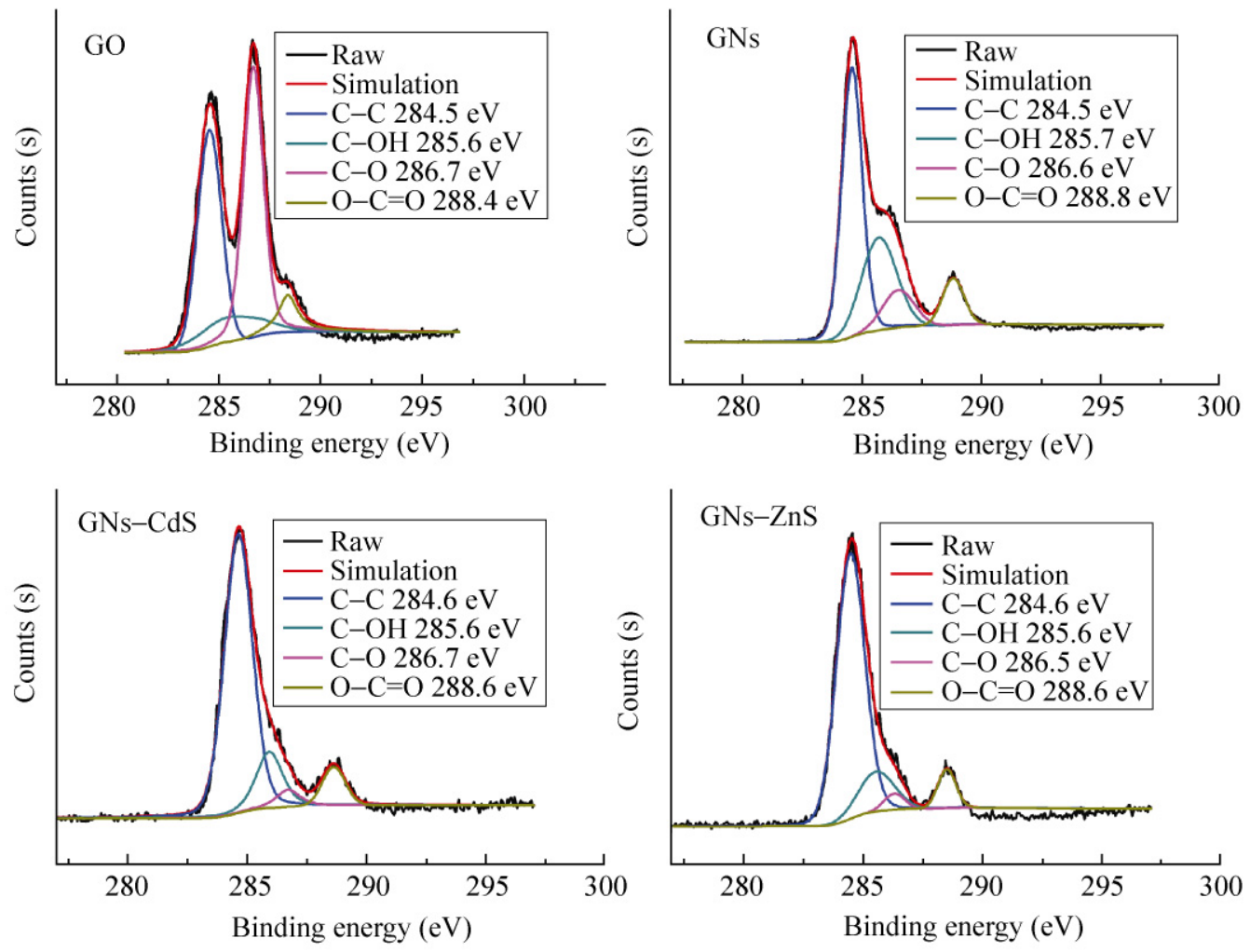

Figure $2 \mathrm{C} 1 \mathrm{~s}$ XPS spectra of GO, GNs, GNs-CdS, and GNs-ZnS 
of GNs $[18,20]$, the GNs-CdS QD system is expected to have great potential for applications in fabricating photovoltaic devices, and further study on the charge transfer properties of the GNs-CdS QD system is thus of interest. We first studied the PL properties of the GNs-CdS QD composite. For comparison, CdS QDs were synthesized via a similar protocol without introducing GO during the reaction. XRD indicates that the crystal phase and size of the CdS QDs are the same as that in the GNs-CdS nanocomposites (Fig. S-5, in the ESM). In the photograph shown in Fig. 3 (inset), it can be clearly seen that under illumination by $365 \mathrm{~nm}$ light, the CdS QDs exhibit strong yellow-green emission, while for the GNs-CdS and GNs, almost no emission could be directly observed with the naked eye. PL measurements (Fig. 3) indicate that after the integration of CdS QDs with GNs, the PL emission of the CdS QDs was significantly quenched. This demonstrates that after the integration, the electron-hole pairs in the excited CdS QDs could be efficiently separated through the injection of electrons from the CdS QDs to GNs, which is consistent with the results observed by Kamat et al [20]. The GNs-ZnS QD nanocomposite exhibits a similar phenomenon (Fig. S-9, in the ESM).

Since the electron-hole pairs in CdS QDs in GNs-CdS are efficiently separated without obvious PL emission, we wondered where the energy of the incident light goes. One possibility is that it contributes to the photovoltaic response; thus, we used the

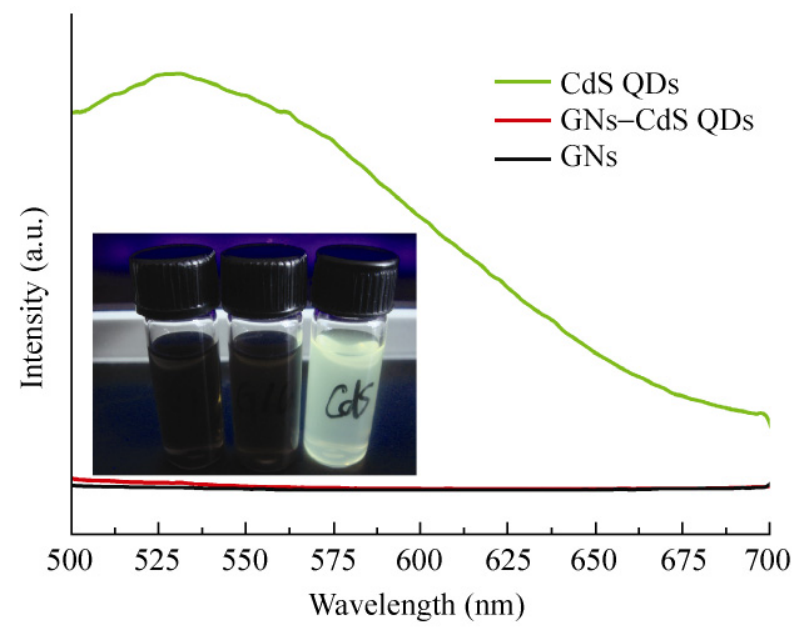

Figure 3 PL spectra of GNs, GNs-CdS, and CdS with an excitation wavelength of $365 \mathrm{~nm}$. The inset shows photographs of the GNs (left), GNs-CdS (middle), and CdS (right) in ethanol under illumination by $365 \mathrm{~nm}$ radiation transient photovoltage (TPV) technique, which is a very promising method for the investigation of dynamic properties of the photo-induced charge carriers in semiconductor materials [21-25], to characterize the GNs-CdS QD system. Figure 4 shows the TPV spectra of the GNs, GNs-CdS, and CdS. Neither GNs nor CdS alone exhibit a photovoltaic response (the very low response is caused by the apparatus). It is wellknown that GNs have metallic character due to their high electron mobility: thus, it is reasonable that there is no photovoltaic response for the pure GNs under illumination by $355 \mathrm{~nm}$ light. As for the CdS QDs, due to their extremely small size (3-4 $\mathrm{nm}$ ), the electron-hole pairs can be considered to be trapped in the QDs, and cannot be efficiently separated, and thus, the material is unable to exhibit an obvious photovoltaic response even when excited in such a strong laser light [26].

Surprisingly, when the CdS QDs are in direct contact with GNs, a very strong positive photovoltaic response can be observed (Fig. 4). This indicates that the GNs have a high ability to accept electrons, and even the electrons generated in such small CdS QDs can overcome the potential barrier of CdS QDs and transfer into GNs, with holes being left in the CdS QDs. This phenomenon can actually be considered as an energy conversion from PL emission to photovoltaic response: for CdS QDs, there is strong PL emission, but without photovoltaic response, while

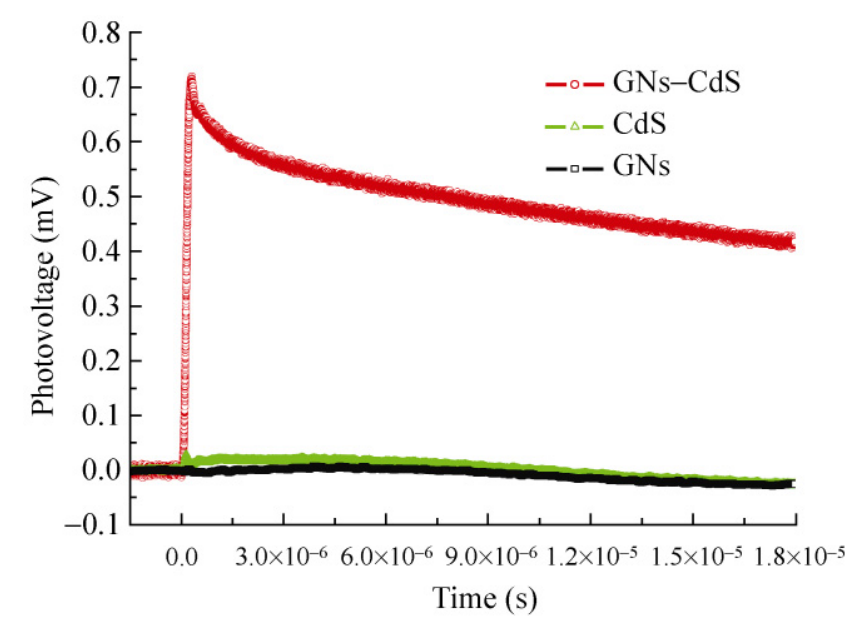

Figure 4 The TPV spectra of GNs, GNs-CdS, and CdS. The wavelength and intensity of the laser were $355 \mathrm{~nm}$ and $0.4 \mathrm{~mJ}$, respectively 
for GNs-CdS, a strong photovoltaic response was observed, but without obvious PL emission.

In conclusion, a facile one-step, solvothermal method for the synthesis of GNs-CdS and GNs-ZnS directly from GO has been developed. The synthesis is very simple and can be extended to synthesize other GNs-metal sulfide (for example PbS-see Figs. S-10 and S-11 in the ESM). Furthermore, the as-synthesized GNs-CdS QDs exhibit very promising photovoltaic properties. The interesting energy conversion phenomenon observed here may provide useful information for developing next-generation high-performance photovoltaic devices.

\section{Acknowledgements}

This work was supported by the National Natural Science Foundation of China (No. 20820102037) and the 973 Project (Nos. 2009CB930100 and 2010CB933600). Dr. Ping Wang gratefully acknowledges partial financial support from the China Postdoctoral Science Foundation (No. 20090461047) and 973 Project (No. 2007CB613303).

Electronic Supplementary Material: Supplementary material (experimental procedures and detailed characterization of products) is available in the online version of this article at http://dx.doi.org/10.1007/s12274$010-0046-0$ and is accessible free of charge.

Open Access: This article is distributed under the terms of the Creative Commons Attribution Noncommercial License which permits any noncommercial use, distribution, and reproduction in any medium, provided the original author(s) and source are credited.

\section{References}

[1] Novoselov, K. S.; Geim, A. K.; Morozov, S. V.; Jiang, D.; Zhang, Y.; Dubonos, S. V.; Grigorieva, I. V.; Firsov, A. A. Electric field effect in atomically thin carbon films. Science 2004, 306, 666-669.

[2] Avouris, P.; Chen, Z.; Perebeinos, V. Carbon-based electronics. Nat. Nanotechnol. 2007, 2, 605-615.

[3] Freitag, M.; Steiner, M.; Martin, Y.; Perebeinos, V.; Chen, Z.; Tsang J. C.; Avouris, P. Energy dissipation in graphene field-effect transistors. Nano Lett. 2009, 9, 1883-1888.

[4] Ang, P. K.; Chen, W.; Wee A. T. S.; Loh, K. P. Solutiongated epitaxial graphene as $\mathrm{pH}$ sensor. J. Am. Chem. Soc. 2008, 130, 14392-14393.

[5] Zhou, M.; Zhai, Y. M.; Dong, S. J. Electrochemical sensing and biosensing platform based on chemically reduced graphene oxide. Anal. Chem. 2009, 81, 5603-5613.

[6] Stoller, M. D.; Park, S.; Zhu, Y.; An J.; Ruoff, R. S. Graphene-based ultracapacitors. Nano Lett. 2008, 8, 34983502.

[7] Stampfer, C.; Schurtenberger, E.; Molitor, F.; Guttinger, J.; Ihn, T.; Ensslin, K. Tunable graphene single electron transistor. Nano Lett. 2008, 8, 2378-2383.

[8] Geim, A. K.; Novoselov, K. S. The rise of graphene. Nat. Mater. 2007, 6, 183-191.

[9] Chae, H. K.; Siberio-Pérez, D. Y.; Kim, J.; Go, Y.; Eddaoudi, M.; Matzger, A. J.; O'Keeffe, M.; Yaghi, O. M. A route to high surface area, porosity and inclusion of large molecules in crystals. Nature 2004, 427, 523-527.

[10] Yong, V.; Tour, J. M. Theoretical efficiency of graphenebased photovoltaics. Small 2010, 6, 313-318.

[11] Guo, C. X.; Yang, H. B.; Sheng, Z. M.; Lu, Z. S.; Song, Q. L.; Li, C. M. Layered graphene/quantum dots for photovoltaic devices. Angew. Chem. Int. Ed. 2010, 49, 3014-3017.

[12] Robel, I.; Bunker, B. A.; Kamat, P. V. Single-walled carbon nanotube-CdS nanocomposites as light-harvesting assemblies: Photoinduced charge-transfer interactions. Adv. Mater. 2005, 17, 2458-2463.

[13] Gu, F.; Li, C.; Wang, S. Solution-chemical synthesis of carbon nanotube/ZnS nanoparticle core/shell heterostructures. Inorg. Chem. 2007, 46, 5343-5348.

[14] Li, F.; Song, J.; Yang, H.; Gan, S.; Zhang, Q.; Han, D.; Ivaska, A.; Niu, L. One-step synthesis of graphene $/ \mathrm{SnO}_{2}$ nanocomposites and its application in electrochemical supercapacitors. Nanotechnology 2009, 20, 455602.

[15] Cao, A.; Liu, Z.; Chu, S.; Wu, M.; Ye, Z.; Cai, Z.; Chang, Y.; Wang, S.; Gong, Q.; Liu, Y. A facile one-step method to produce graphene-CdS quantum dot nanocomposites as promising optoelectronic materials. Adv. Mater. 2010, 22, 103-106.

[16] Paredes, J. I.; Villar-Rodil, S.; Martinez-Alonso, A.; Tascon, J. M. D. Graphene oxide dispersions in organic solvents. Langmuir 2008, 24, 10560-10564.

[17] Nethravathi, C.; Rajamathi, M. Chemically modified graphene sheets produced by the solvothermal reduction of colloidal dispersions of graphite oxide. Carbon 2008, 46, 1994-1998.

[18] Williams, G.; Seger, B.; Kamat, P. V. TiO ${ }_{2}$-graphene nanocomposites. UV-assisted photocatalytic reduction of graphene oxide. ACS Nano 2008, 2, 1487-1491. 
[19] Yuge, R.; Zhang, M.; Tomonari, M.; Yoshitake, T.; Iijima, S.; Yudasaka, M. Site identification of carboxyl groups on graphene edges with $\mathrm{Pt}$ derivatives. ACS Nano 2008, 2, 1865-1870.

[20] Williams, G.; Kamat, P. V. Graphene-semiconductor nanocomposites: Excited-state interactions between $\mathrm{ZnO}$ nanoparticles and graphene oxide. Langmuir 2009, 25, 13869-13873.

[21] Duzhko, V.; Timoshenko, V. Y.; Koch, F.; Dittrich, T. Photovoltage in nanocrystalline porous $\mathrm{TiO}_{2}$. Phys. Rev. $B$ 2001, 64, 075204.

[22] Fang, Q.; Zhu, G.; Jin, Z.; Xue, M.; Wei, X.; Wang, D.; Qiu, S. A multifunctional metal-organic open framework with a bcu topology constructed from undecanuclear clusters. Angew. Chem. Int. Ed. 2006, 45, 6126-6130.

[23] Wei, X.; Xie, T.; Xu, D.; Zhao, Q.; Pang, S.; Wang, D. A study of the dynamic properties of photo-induced charge carriers at nanoporous $\mathrm{TiO}_{2}$ /conductive substrate interfaces by the transient photovoltage technique. Nanotechnology 2008, 19, 275707.

[24] Wang, P.; Xie, T.; Li, H.; Peng, L.; Zhang, Y.; Wu, T.; Pang, S.; Zhao, Y.; Wang, D. Synthesis and plasmon-induced charge-transfer properties of monodisperse gold-doped titania microspheres. Chem. Eur. J. 2009, 15, 4366-4372.

[25] Zhang, Q.; Wang, D.; Wei, X.; Xie, T.; Li, Z.; Lin, Y.; Yang, M. A study of the interface and the related electronic properties in $n-\mathrm{Al}_{0.35} \mathrm{Ga}_{0.65} \mathrm{~N} / \mathrm{GaN}$ heterostructure. Thin Solid Films 2005, 491, 242-248.

[26] Lin, Y.; Wang, D.; Zhao, Q.; Yang, M.; Zhang, Q. A study of quantum confinement properties of photogenerated charges in $\mathrm{ZnO}$ nanoparticles by surface photovoltage spectroscopy. J. Phys. Chem. B 2004, 108, 3202-3206. 